\title{
Outcome of In-Hospital Cardiac Arrest in Adult General Wards
}

\author{
Chia-Te Kung1,2, Hsien-Hung Cheng1,2, Shin-Chiang Hung1,2, Chao-Jui Li1,2, Chu-Feng Liu1,2, \\ Fu-Cheng Chen',2, Chih-Min Su',2, Jien-Wei Liu ${ }^{2,3}$, Hung-Yi Chuang4,5* \\ ${ }^{1}$ Department of Emergency Medicine, Kaohsiung Chang Gung Memorial Hospital, Kaohsiung, Taiwan \\ ${ }^{2}$ Chang Gung University College of Medicine, Kaohsiung, Taiwan \\ ${ }^{3}$ Department of Infection, Kaohsiung Chang Gung Memorial Hospital, Kaohsiung, Taiwan \\ ${ }^{4}$ Department of Community Medicine, Kaohsiung Medical University Hospital, Kaohsiung City, Taiwan \\ ${ }^{5}$ Department of Public Health, College of Health Sciences, Kaohsiung Medical University, Kaohsiung City, \\ Taiwan \\ Email: chaio1130@gmail.com, ${ }^{*}$ kungchiate@gmail.com
}

Received 23 August 2014; revised 22 September 2014; accepted 20 October 2014

Copyright (C) 2014 by authors and Scientific Research Publishing Inc.

This work is licensed under the Creative Commons Attribution International License (CC BY). http://creativecommons.org/licenses/by/4.0/

cC) (7) Open Access

\begin{abstract}
Objective: Few studies have focused on factors influencing outcomes of patients with in-hospital cardiac arrest (IHCA) in general wards. The goal of this study was to report the outcomes of adult patients with IHCA in the general wards and identified the prognostic factors. Methods: Adult patients with IHCA having received cardiopulmonary resuscitation in general wards from January 2008 to December 2011 were retrospectively reviewed from our registry system. The primary outcome was survival to hospital discharge, while the secondary outcome was sustained return of spontaneous circulation (ROSC). Results: A total of 544 general ward patients were analyzed for event variables and resuscitation results. The rate of establishing a ROSC was $40.1 \%$ and the rate of survival to discharge was $5.1 \%$. Ventricular tachycardia/ventricular fibrillation (VT/VF) was the initial rhythm in $3.9 \%$ of patients. Pre-arrest factors including a high Charlson comorbidity index (CCI) $\geq 9$ (OR 0.251, 95\% CI 0.098 - 0.646), cardiac comorbidity (OR 0.612, 95\% CI 0.401 0.933), and arrest time on the midnight shift (OR 0.403, 95\% CI $0.252-0.642$ ) were independently associated with a low possibility of ROSC. The initial VT/VF presenting rhythms (OR $0.135,95 \%$ CI $0.030-0.601$ ) were independently associated with a high survival rate, whereas patients with deteriorated disease course were independently associated with a decreased hospital survival (OR $3.902,95 \%$ CI 1.619 - 9.403). Conclusions: We demonstrated that pre-arrest factors can predict patient outcome after IHCA in general wards, including the association of a CCI $\geq 9$ and cardiac comorbidity with poor ROSC, and deteriorated disease course as an independent predictor of a low survival rate.
\end{abstract}

${ }^{*}$ Corresponding author.

How to cite this paper: Kung, C.-T., Cheng, H.-H., Hung, S.-C., Li, C.-J., Liu, C.,-F., Chen, F.-C., Su, C.-M., Liu, J.-W. and Chuang, H.-Y. (2014) Outcome of In-Hospital Cardiac Arrest in Adult General Wards. International Journal of Clinical Medicine, 5, 1228-1237. http://dx.doi.org/10.4236/ijcm.2014.519157 


\section{Keywords}

\section{In-Hospital Cardiac Arrest, Charlson Comorbidity Index, General Ward, Cardiopulmonary Resuscitation, Outcome}

\section{Introduction}

According to the Get with the Guidelines (GWTG)-Resuscitation registry, overall survival after in-hospital cardiac arrest (IHCA) has improved significantly during the past decade [1] [2]. Survival to discharge rates increased from $13.7 \%$ in 2000 to $22.3 \%$ in 2009. The most important explanations for the apparent improvement are advances in the management of acute myocardial infarction and heart failure that may have led to a decline in the proportion of IHCA of which the initial rhythm is ventricular fibrillation (VF) or pulseless ventricular tachycardia (VT) [1]-[4]. Therefore, a good outcome is associated with cardiac morbidity, the event being witnessed, and the patient being monitored [3]-[8]. However, the patient outcome after IHCA in general ward remains poor with only $3 \%-14 \%$ of patients undergoing cardiopulmonary resuscitation (CPR) being able to survive and be discharged [3] [9] [10]. Previous reports indicated that patients with cardiac arrest in critical care areas had a better outcome than those in general wards because of different policies, manpower, disease entities, staff training and experience in response to emergency situations [5]-[11]. Established outcome predictors for patients with IHCA such as adequate monitoring may not be easily applied to those in the general wards. To our knowledge, few studies have focused on factors influencing outcome of patients with IHCA in general wards [9] [10]. The goal of this study was to report the outcomes of adult patients with IHCA in the general wards of an Asian medical center in comparison to those in western countries.

\section{Material and Methods}

\subsection{Study Design and Hospital Setting}

The study was conducted as a retrospective analysis of a dedicated cardiac arrest registry containing prospectively collected data on IHCA at the Kaohsiung Chang Gung Memorial Hospital (CGMH) from January 2008 to December 2011. Kaohsiung CGMH is a tertiary teaching hospital, with 2715 beds including 1388 in adult general ward and 203 in adult intensive care unit (ICU). The hospital's Institutional Review Committee on Human Research approved the study protocol.

\subsection{Enrollment of Participants}

Adult patients ( $\geq 18$ years of age) at the time of hospital admission who received chest compression and/or defibrillation and advanced airway management in general wards were included in the study. Exclusion criteria included1) outpatients; 2) patients in emergency department/ICU/coronary care unit/operating rooms; 3) patients receiving only resuscitation drugs or positive pressure ventilation without chest compression or defibrillation, 4) patients subject to palliative treatment or to a "do not resuscitate (DNR)" order; 5) patients experiencing a second or subsequent cardiac arrest; and 6) patients with pre-existing tracheostomy or mechanical ventilation.

\subsection{Management of Cardiac Arrest}

In addition to the physicians and nurses on duty in the wards, there is an emergency response team at the institute that provides round-the-clock CPR for those in need. All members of the resuscitation team were certified in advanced cardiac life support (ACLS). Additionally, there was a dedicated nurse who recorded all of the resuscitation efforts, procedures, and medications on a standardized resuscitation data form during the event. We used the Utstein definitions and recommendations to record the demographic data, event and outcome variables of all patients studied [12]. During the study period, no therapeutic hypothermia and extracorporeal membrane oxygenation support was performed during CPR or in the post-resuscitative period in our general wards.

\subsection{Assessment of Patient Outcomes}

The primary parameter for the assessment of patient outcome was survival to hospital discharge. Parameters for 
secondary outcome included return of spontaneous circulation (ROSC) for more than 20 minutes with no further need for chest compressions. Neurological outcome was determined using Cerebral Performance Category (CPC) score which allocates a score of 1 for good cerebral performance, 2 for moderate performance, 3 for poor performance, 4 for comatose or vegetative status, and 5 for brain death. In this study, patients with a score of one or two were defined as those with favorable neurological outcomes.

\subsection{Data Collection and Definition}

Independent variables comprised those in the pre-arrest, peri-arrest, and intra-arrest settings. Pre-arrest variables included basic patient characteristics, comorbidities before admission, main morbidity on admission, and intervention on admission. Peri-arrest variables were time of arrest, admitting department, interventions performed during the initial hospitalization period in wards before arrest. Intra-arrest variables included the first documented pulseless rhythm, witness of the event, the time interval from patient collapse to the beginning of CPR, and that from patient collapse to ROSC.

A deteriorated disease course was defined as a respiratory rate of $\geq 32$ breaths/min or $\leq 5$ breaths/min, a pulse rate of $\leq 40$ beats/min or $\geq 140$ beats/min, a systolic blood pressure of $<90 \mathrm{mmHg}$, and a sudden fall in the level of consciousness by 2 or more Glasgow Coma Scale points within 8 hours before cardiac arrest [13].

\subsection{Data Analysis}

Frequencies were generated for independent variables and outcomes. Categorical variables were evaluated using Chi-square tests or Fisher's exact test. Continuous variables were expressed as mean \pm standard deviation (SD) or median with interquartile range, and were analyzed with a two-tailed Student's t-test or Mann-Whitney U-test for independent variables. Statistical significance was defined as $p<0.05$. Univariate analyses were first performed to select potential risk factors. Variables significantly associated with ROSC and hospital discharge ( $p<$ 0.05 ) in univariate analysis and patient characteristics (age and functional status before hospital admission) were entered into a multivariate logistic regression for the analysis of both primary and secondary outcome. Results were considered significant if $p<0.05$. All analyses were performed using SPSS version 14.0 (SPSS Inc., Chicago, IL, USA).

\section{Results}

\subsection{Patients and Event Characteristics}

During the study period, a total of 544 adult patients with IHCA occurring in general wards were analyzed for the event variables and resuscitation results (Figure 1). Of the 544 patients, 218 (40.1\%) had ROSC, only 28 (5.1\%) survived to hospital discharge, and 6 (1.1\%) were discharged with favorable neurological outcomes.

Patient demographics are shown in Table 1. The mean age was $67.8 \pm 16.2$ years (interquartile range: 58 - 79 years) and $58.8 \%$ were men. Univariate analysis showed significant correlation between the ROSC rate and Charlson comorbidity index (CCI) score $(p=0.014)$. The initial rhythms recorded when the event took place were pulseless electrical activity (PEA) for over half of the patients (61.7\%), while VT/VF was recorded in only $3.9 \%$ of patients. When VT/VF was documented as the first rhythm, patients had a higher rate of ROSC compared to those with initial presentations of PEA and asystole (52.4\% vs. $43.8 \%$ vs. $32.1 \%, p=0.017)$ and survival to discharge $(19.0 \%$ vs. $5.4 \%$ vs. $3.2 \%, p=0.008)$. Patients with witnessed arrest were more likely to achieve ROSC compared to those with unwitnessed arrest $(44.6 \%$ vs. $31.0 \%, p=0.006)$, whereas there was no significant difference between survival to hospital discharge between the two groups. Most of the arrest events (39.2\%) occurred during the midnight shift (00:00-07:59). There was a significant difference between the time of arrest and ROSC (day shift $49.7 \%$ vs. night shift $47.4 \%$ vs. midnight shift $26.8 \%, p<0.001$ ). The majority of patients (54.6\%) in this study had a deteriorated disease course before arrest that was significantly associated with a poorer discharge outcome compared to those without a deteriorated course $(p=0.005)$.

\subsection{Morbidities before and on Admission}

Morbidities before admission are shown in Figure 2. Coronary artery disease accounted for the underlying etiology of cardiac arrest in 81 (14.8\%) patients, while cancer and hematological malignancy are the underlying 


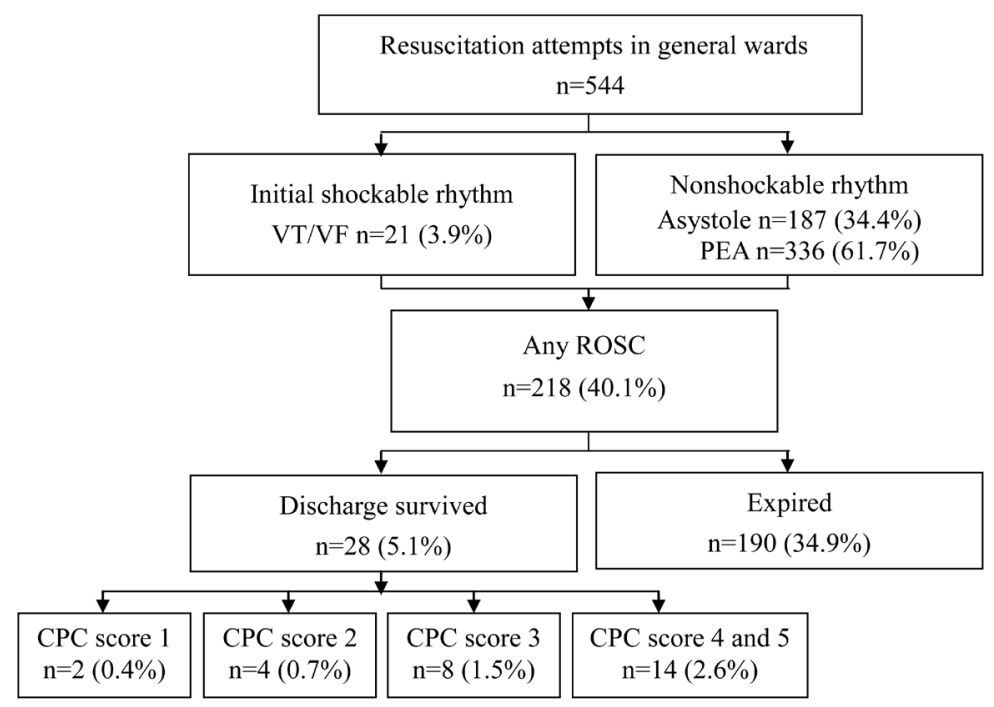

Figure 1. Cardiopulmonary arrest events in general wards. $\mathrm{CPC}=$ cerebral performance category; PEA = pulseless electrical activity; ROSC = return of spontaneous circulation; VF = ventricular fibrillation; VT = ventricular tachycardia.

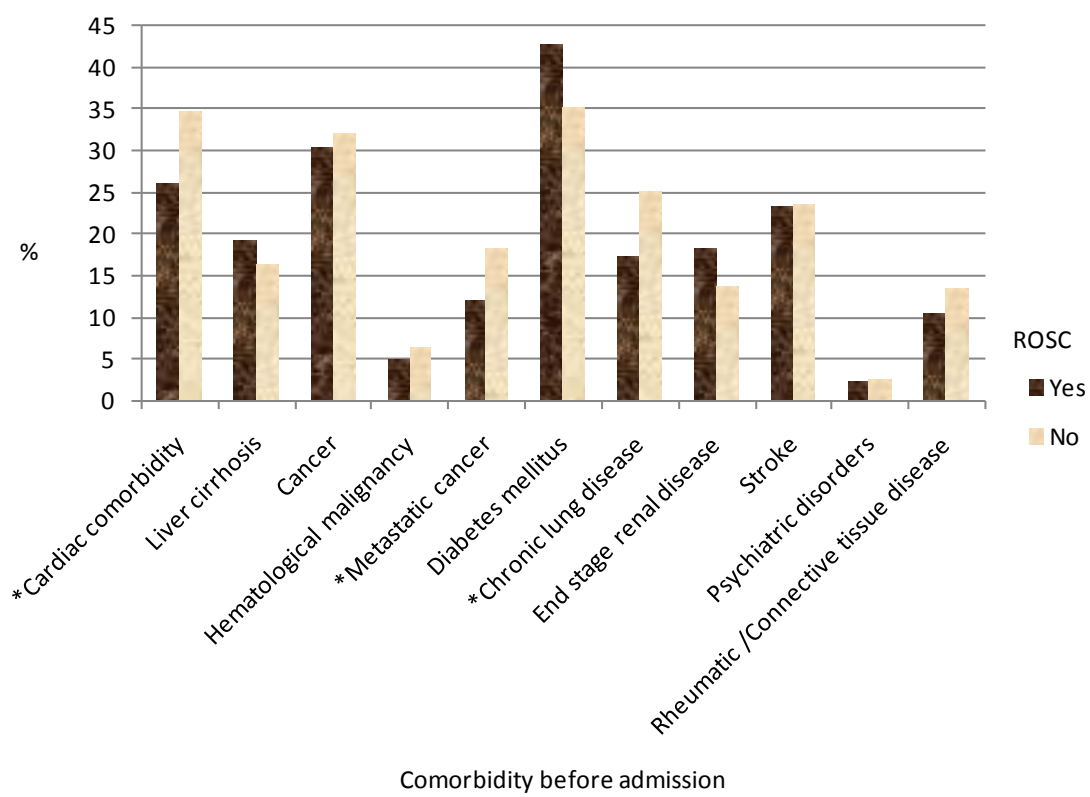

Figure 2. Comorbidity before admission with ROSC. ROSC = return of spontaneous circulation. ${ }^{*} p<0.05$, ROSC vs. non-ROSC.

morbidities in $37.3 \%$ of the patients. Patients with cardiac morbidity before admission had reduced chances of ROSC compared with those without $(p=0.036)$. Also, patients with chronic lung disease and metastatic cancer before admission were less likely to achieve ROSC compared to those without ( $31.7 \%$ vs. $42.5 \%, p=0.033$ and $30.2 \%$ vs. $41.9 \%, p=0.042$, respectively) (Figure 2). Patients with diabetes mellitus before admission had higher rate of survival to discharge $(57.1 \%$ vs. $37.2 \%, p=0.046)$ (Figure 3).

Cancer treatment or complications from cancer (18.9\%) and pneumonia/chronic lung diseases (16.9\%) were the two most common admission diagnoses. Cardiac morbidity was found in 46 (8.5\%) patients, including 15 (2.8\%) patients with acute myocardial infarction on admission. However, there was no relation between admission morbidities and ROSC or discharge outcome. 
Table 1. Patient demographics and event characteristics.

\begin{tabular}{|c|c|c|c|c|c|c|}
\hline \multirow{2}{*}{ Variables } & \multicolumn{3}{|c|}{ ROSC } & \multicolumn{3}{|c|}{ Discharge survived } \\
\hline & Yes (218) & No (326) & $p$ & Yes (28) & No (516) & $p$ \\
\hline \multicolumn{7}{|l|}{ Demographic characteristics } \\
\hline Male sex & $121(55.5)$ & 199 (61.0) & 0.198 & $15(53.6)$ & $305(59.1)$ & 0.562 \\
\hline Age & & & 0.888 & & & 0.548 \\
\hline $18-40$ & $11(5.0)$ & $20(6.1)$ & & $3(10.7)$ & $28(5.4)$ & \\
\hline $41-65$ & $78(35.8)$ & $108(33.1)$ & & $7(25.0)$ & $179(34.7)$ & \\
\hline $66-79$ & $79(36.2)$ & 119 (36.5) & & $11(39.3)$ & $187(36.2)$ & \\
\hline$\geq 80$ & $50(22.9)$ & $79(24.2)$ & & $7(25.0)$ & $122(23.6)$ & \\
\hline BMI & & & 0.745 & & & 0.083 \\
\hline$\leq 18.5$ & $30(15.2)$ & $41(13.7)$ & & $4(16.7)$ & $67(14.2)$ & \\
\hline $18.6-24.9$ & $103(52.3)$ & $171(57.0)$ & & $9(37.5)$ & $265(56.0)$ & \\
\hline $25-29.9$ & $53(26.9)$ & $75(25.0)$ & & $11(45.8)$ & $117(24.7)$ & \\
\hline$\geq 30$ & $11(5.6)$ & $13(4.3)$ & & $0(0)$ & $24(5.1)$ & \\
\hline Residence before admission & & & 0.657 & & & 0.584 \\
\hline Home & $175(80.3)$ & $253(77.6)$ & & $23(82.1)$ & 405 (78.5) & \\
\hline Another hospital & $37(17.0)$ & $60(18.4)$ & & $5(17.9)$ & $92(17.8)$ & \\
\hline Nursing home & $6(2.8)$ & $13(4.0)$ & & $0(0)$ & $19(3.7)$ & \\
\hline Admission CPC & & & 0.592 & & & 0.917 \\
\hline 1 & $98(45.0)$ & $132(40.5)$ & & $12(42.9)$ & $218(42.2)$ & \\
\hline 2 & $94(43.1)$ & 143 (43.9) & & $13(46.5)$ & $224(43.4)$ & \\
\hline 3 & $24(11.0)$ & $47(14.4)$ & & $3(10.7)$ & $68(13.2)$ & \\
\hline 4 & $2(0.9)$ & $4(1.2)$ & & $0(0)$ & $6(1.2)$ & \\
\hline Admission site & & & 0.639 & & & 0.677 \\
\hline ED & $196(89.9)$ & $297(91.1)$ & & $26(92.9)$ & 467 (90.5) & \\
\hline OPD & $22(10.1)$ & $29(8.9)$ & & $2(7.1)$ & $49(9.5)$ & \\
\hline Charlson comorbidity index & & & 0.014 & & & 0.575 \\
\hline 0 & $7(3.2)$ & $12(3.7)$ & & $1(3.6)$ & $18(3.5)$ & \\
\hline $1-3$ & 69 (31.7) & $102(31.3)$ & & $12(42.9)$ & $159(30.8)$ & \\
\hline $4-6$ & $106(48.6)$ & $136(41.7)$ & & $10(35.7)$ & $232(45.0)$ & \\
\hline $7-9$ & $35(16.1)$ & $56(17.2)$ & & $5(17.9)$ & $86(16.7)$ & \\
\hline$>9$ & $1(0.5)$ & $20(6.1)$ & & $0(0)$ & $21(4.1)$ & \\
\hline Total number of comorbidities & & & 0.965 & & & 0.687 \\
\hline 0 & $3(1.4)$ & $4(1.2)$ & & $0(0)$ & $7(1.4)$ & \\
\hline $1-3$ & $126(57.8)$ & 195 (59.8) & & $15(53.6)$ & $306(59.3)$ & \\
\hline $4-6$ & $86(39.4)$ & $122(37.4)$ & & $13(46.4)$ & $195(37.8)$ & \\
\hline$>6$ & $3(1.4)$ & $5(1.5)$ & & $0(0)$ & $8(1.6)$ & \\
\hline \multicolumn{7}{|l|}{ Characteristics of cardiac arrest } \\
\hline First documented rhythm & & & 0.017 & & & 0.02 \\
\hline Asystol & $60(27.5)$ & $127(39.0)$ & & $6(21.4)$ & $181(35.1)$ & \\
\hline $\mathrm{VF} / \mathrm{VT}$ & $11(5.0)$ & $10(3.1)$ & & $4(14.3)$ & $17(3.3)$ & \\
\hline PEA & $147(67.4)$ & $189(58.0)$ & & $18(64.3)$ & 318 (61.6) & \\
\hline Witness arrest & $158(72.5)$ & $196(60.1)$ & 0.006 & $17(60.7)$ & 337 (65.3) & 0.796 \\
\hline
\end{tabular}




\section{Continued}

\begin{tabular}{|c|c|c|c|c|c|c|}
\hline People who found arrest & & & 0.094 & & & 0.436 \\
\hline Staff & $143(65.6)$ & $223(68.4)$ & & $16(57.1)$ & $350(67.8)$ & \\
\hline Family & $75(34.4)$ & $103(31.6)$ & & $12(42.9)$ & $166(32.2)$ & \\
\hline Deteriorated disease course & $120(55.0)$ & 177 (54.3) & 0.863 & $8(28.6)$ & $289(56.0)$ & 0.005 \\
\hline Admission department & & & 0.066 & & & 0.079 \\
\hline Medical & $195(89.4)$ & $295(90.5)$ & & $23(82.1)$ & 467 (90.5) & \\
\hline Surgical & $21(9.6)$ & $20(6.1)$ & & 5 (17.9) & $36(7.0)$ & \\
\hline Others & $2(0.9)$ & $11(3.4)$ & & $0(0)$ & $13(2.5)$ & \\
\hline Time of arrest & & & $<0.001$ & & & 0.480 \\
\hline Day shift (8:00-15:59) & 87 (39.9) & $88(27.0)$ & & $10(35.7)$ & $165(32.0)$ & \\
\hline Night shift (16:00-23:59) & 74 (33.9) & $82(25.2)$ & & $10(35.7)$ & $146(28.3)$ & \\
\hline Midnight shift (0:00-07:59) & $57(26.1)$ & $156(47.9)$ & & $8(28.6)$ & 205 (39.7) & \\
\hline
\end{tabular}

Values are presented as number (\%). ROSC = return of spontaneous circulation; BMI = body mass index; CPC = cerebral performance category; ED = emergency department; OPD = outpatient department; VF = ventricular fibrillation; VT = ventricular tachycardia; PEA = pulseless electrical activity.

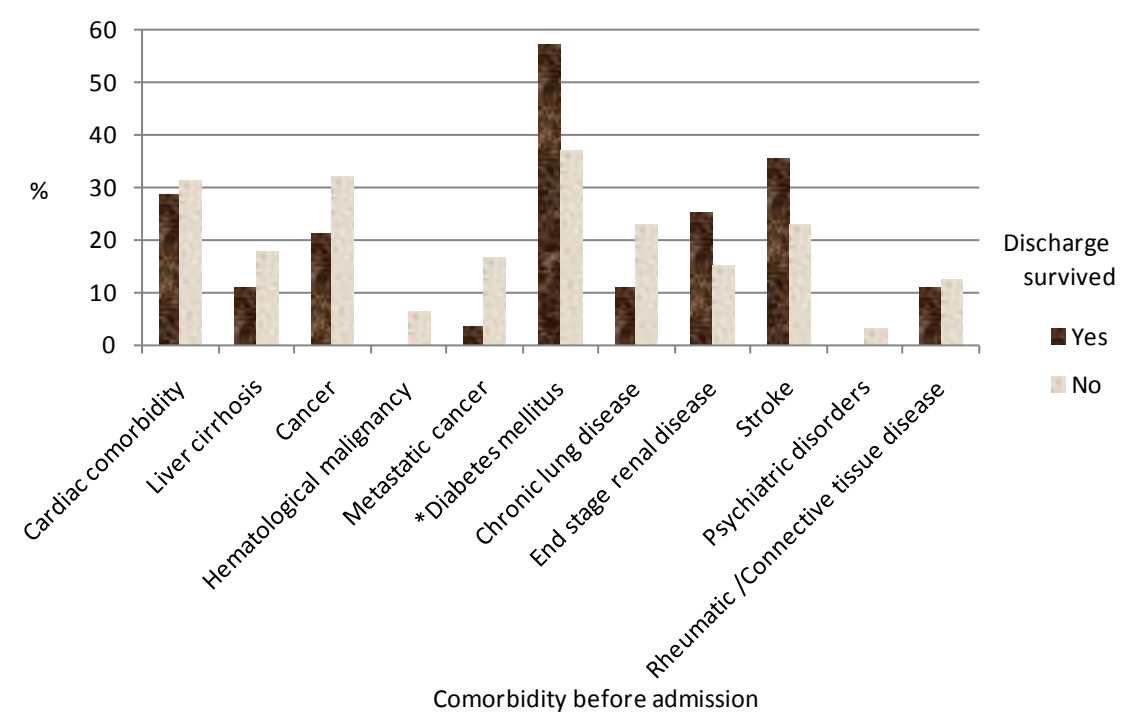

Figure 3. Comorbidity before admission with discharge outcome. ${ }^{*} p<0.05$, discharge survivors vs. discharge non-survivors.

\subsection{Outcome of Cardiopulmonary Arrest Events}

The mean CPR duration in the ROSC group was $21.2 \pm 11.3$ minutes (range $3-75$ minutes). The mean period of hospitalization in the survival group was significantly longer than that of the mortality group (98.75 \pm 87.13 vs. $21.84 \pm 39.67$ days, $p<0.001)$. We found that the probability of successful resuscitation $\left(p_{\text {trend }}=0.027\right)$ and survival to discharge $\left(p_{\text {trend }}=0.036\right)$ decreased as the CCI score increased. In this study, we also identified 5 pre-arrest conditions indicative of an extremely poor prognosis with $100 \%$ mortality after IHCA events, including a body mass index $\geq 30$ ( 24 patients), a high CCI score $\geq 9$ (36 patients), as well as the underlying diseases of myocardial infarction (22 patients), hematological malignancy (32 patients), and admission diagnoses of cirrhosis-related complications (44 patients).

\subsection{Multivariate Regression Models}

Four factors were independently associated with ROSC. A high CCI score $\geq 9$, cardiac morbidity, metastatic malignancy before admission, and arrest during the midnight shift were independently associated with a low 
possibility of ROSC (Table 2). An initial presentation of VT/VF pulseless rhythm was independently associated with a high survival rate, whereas a deteriorated disease course was independently associated with a low possibility of survival to hospital discharge (Table 3).

\section{Discussion}

We describe the outcomes of CPR for patients with IHCA in general wards. The overall patient survival was $5.1 \%$, while $1.1 \%$ of all patients showed favorable neurological outcomes. The findings of the current study demonstrated several previously unreported factors that had significant impact on the survival of patients with IHCA in the pre-arrest setting. For instance, a reduced chance of ROSC was noted in the presence of a CCI $\geq 9$. Moreover, the probability of successful resuscitation and survival to discharge decreased as the CCI score increased. Furthermore, cardiac comorbidity before admission was independently associated with a low possibility of ROSC. Finally, a deteriorated disease course was independently associated with an unfavorable patient survival.

According to the national registry of cardiopulmonary resuscitation (NRCPR), patients who underwent CPR in general inpatient area had a 14\% probability of survival to discharge [3]. The majority of studies report better outcomes for patient with IHCA occurring in critical care areas than those encountered in wards [3] [5] [6] [11]. The possible explanations included the monitored and witnessed status of the patients, immediate availability of personnel and medications for conducting effective ACLS, and the nature of patients in a critical care setting. For instance, patients with cardiac morbidity in the coronary care unit are likely to develop cardiac arrests secondary to readily reversible dysrhythmias that may contribute to a relatively good prognosis. The data from NRCPR showed that $77.7 \%$ of overall patients were being monitored without specifically referring to patients in the general wards [3]. By contrast, in our general ward population, only $16 \%$ of patients were being monitored before arrest, even though $54.6 \%$ of patients had abnormal vital signs according to the definition of deteriorated disease courses. The inherent limitations of general wards and inadequate monitoring, therefore, may be a contributor to unfavorable patient outcome.

Cardiac arrest occurs in a heterogeneous group of patients commonly hospitalized for serious comorbidities. Indeed, the data of GWTG-Resuscitation registry showed that the absence of renal insufficiency, hepatic insufficiency, sepsis, and malignancy prior to the arrest were associated with favorable neurological survival [5]. In this study, CCI and the total number of comorbidities before admission were used as the indices of severity to evaluate the correlation between comorbidities before admission and patient outcome. However, the present study demonstrated no significant association between patient survival and the two indices with the exception of a reduced probability of ROSC in the presence of a CCI $\geq 9$. On the other hand, stratification of the data with a more detailed CCI showed a trend indicative of an association of decreased probability of successful resuscitation and discharge survival with an elevated CCI. Although a high CCI was not a significant discharge outcome predictor in this study, all 36 patients with a CCI $\geq 9$ did not survive to hospital discharge. The very low survival

Table 2. Factors independently associated with return of spontaneous circulation, using multivariate analysis.

\begin{tabular}{ccc}
\hline Factor & Odds ratio (95\% CI) & $p$ \\
\hline Charlson comorbidity index $\geq 9$ & $0.251(0.098-0.646)$ & 0.004 \\
Cardiac comorbidity before admission & $0.612(0.401-0.933)$ & 0.023 \\
Metastatic malignancy before admission & $0.485(0.282-0.835)$ & 0.009 \\
Arrest time at midnight shift (0:00-07:59) & $0.403(0.252-0.642)$ & $<0.001$ \\
\hline
\end{tabular}

Table 3. Factors independently associated with in-hospital mortality, using multivariate analysis.

\begin{tabular}{ccc} 
Factor & Odds ratio (95\% CI) & $p$ \\
\hline Deteriorated disease course & $3.902(1.619-9.403)$ & 0.002 \\
Initial rhythm with VT/VF & $0.135(0.030-0.601)$ & 0.009 \\
\hline
\end{tabular}

$\mathrm{VF}=$ ventricular fibrillation; $\mathrm{VT}=$ ventricular tachycardia. 
rate may account for its lack of significance as a predictor. Although data from NRCPR suggested that nine preexisting conditions and the mean number of pre-existing conditions were significantly related to the discharge mortality risk strata [3], there was no significant relationship between patient survival and the total number of comorbidities before admission in our study. CCI, which is constructed by assigning a weight to each comorbidity according to the magnitude of the relative risk associated with each condition [14] [15], has not been previously reported as an outcome predictor of IHCA. In comparison with the assessment of risk associated with a specific disease or the total number of comorbidities, CCI is a more reliable indicator for outcome prediction after IHCA in the pre-arrest setting.

The NRCPR study and that by de Vos et al. revealed that patients with cardiac morbidity had a nearly twofold increase in survival rate compared with those with non-cardiac morbidity [3] [16]. However, the results of the current study shows that cardiac morbidity was significantly associated with a poorer ROSC rate, contrary to those of previous studies that demonstrated significant association of cardiac morbidity with a higher probability of ROSC and survival rate. In addition to the event locations and inadequate monitoring, another difference of our study from previous reports is the lower prevalence of coronary artery disease at admission (14.8\%) in our study population. Previous studies have reported higher prevalence of ischemic heart disease in western countries. Ballew et al. demonstrated that $43 \%$ of the study population had coronary artery disease and $31 \%$ had a previous myocardial infarction [17]. Herlitz et al. also revealed that $42 \%$ of men and $25 \%$ of women with IHCA had a previous history of myocardial infarction [18]. The relatively low frequency of ischemic heart disease in this study is comparable to that of an out-of-hospital cardiac arrest study in Japan [19]. Accordingly, it also accounted for the very low incidence (3.9\%) of VT/VF as the first documented rhythm in this study. Taken together, it may account for our finding that cardiac morbidity was not a positive predictor for ROSC or survival to hospital discharge in the general wards. On the other hand, according to the data of NRCPR, another significant factor associated with a decreased survival of adult patients with IHCA is resuscitation system errors that has been reported to occur most frequently (up to $40.4 \%$ ) in non-ICU inpatient areas [20]. In general wards, a lack of manpower, inadequate patient monitorings, late discovery of arrests, or delayed response of the CPR team could occur especially at midnight shift, thereby missing the chance of timely treatment of reversible rhythms such as VT/VF.

Previous reports indicated that the majority (i.e., approximately 50\% to 84\%) of patients had identifiable serious physiological abnormalities within eight hours preceding IHCA [13] [21] [22]. In our study, 54.6\% of patients had abnormal vital signs according to the definition of deteriorated disease course. We also identified five pre-arrest conditions indicative of an extremely poor prognosis with $100 \%$ mortality after IHCA episodes in this study. For these high risk patients, placing DNR discussions is of paramount importance in such deterioration conditions. Patients who are unlikely to benefit from CPR should be identified on or during hospital admission and the possibility of DNR orders should be discussed to avoid inappropriate treatment and potential patient suffering.

This study had its limitations. First, our study suffered from the shortcomings of the setting of a single institute and its retrospective nature including inadequate documentation at the time of CPR and incomplete or missing patient records. Second, the sample size of our survival group was relatively small. Finally, the long period covered in this study may have introduced significant confounders that influenced the outcomes.

\section{Conclusion}

The overall survival for patients with IHCA in general wards remained poor. Reduced chance of ROSC was noted in the presence of a CCI $\geq 9$ as well as the presence of metastatic malignancy and cardiac comorbidity before admission. Moreover, the probability of successful resuscitation and survival to discharge also decreased as CCI score increased. Furthermore, a deteriorated disease course was independently associated with an unfavorable patient survival.

\section{Authors' Contributions}

CTK participated in the design of the study and drafted the manuscript. HHC, SCH, CFL, FCC, and CMS participated in the coordination of the study and data collection. CJL, HYC and HHC performed the statistical analysis and interpretation of statistical data. JWL and HYC conceived the study, participated in its design and coordination, and helped draft the manuscript. All authors read and approved the final manuscript. 


\section{References}

[1] Girotra, S., Nallamothu, B.K., Spertus, J.A., Spertus, J.A., Li, Y., Krumholz, H.M. and Chan, P.S. (2012) Trends in Survival after In-Hospital Cardiac Arrest. New England Journal of Medicine, 367, 1912-1920. http://dx.doi.org/10.1056/NEJMoa1109148

[2] Girotra, S., Cram, P., Spertus, J.A., Nallamothu, B.K., Li, Y., Jones, P.G. and Chan, P.S. (2014) Hospital Variation in Survival Trends for In-Hospital Cardiac Arrest. Journal of the American Heart Association, 3, e000871. http://jaha.ahajournals.org/content/3/3/e000871.long http://dx.doi.org/10.1161/JAHA.114.000871

[3] Meaney, P.A., Nadkarni, V.M., Kern, K.B., Indik, J.H., Halperin, H.R. and Berg, R.A. (2010) Rhythms and Outcomes of Adult In-Hospital Cardiac Arrest. Critical Care Medicine, 38, 101-108. http://dx.doi.org/10.1097/CCM.0b013e3181b43282

[4] Larkin, G.L., Copes, W.S., Nathanson, B.H., Larkin, G.L., Copes, W.S., Nathanson, B.H. and Kaye, W. (2010) PreResuscitation Factors Associated with Mortality in 49,130 Cases of In-Hospital Cardiac Arrest: A Report from the National Registry for Cardiopulmonary Resuscitation. Resuscitation, 81, 302-311. http://dx.doi.org/10.1016/j.resuscitation.2009.11.021

[5] Chan, P.S., Spertus, J.A., Krumholz, H.M., Berg, R.A., Li, Y., Sasson, C. and Nallamothu, B.K. (2012) A Validated Prediction Tool for Initial Survivors of In-Hospital Cardiac Arrest. Archives of Internal Medicine, 172, 947-953. http://dx.doi.org/10.1001/archinternmed.2012.2050

[6] Brady, W.J., Gurka, K.K., Mehring, B., Peberdy, M.A. and O’Connor, R.E. (2011) In-Hospital Cardiac Arrest: Impact of Monitoring and Witnessed Event on Patient Arrival and Neurologic Status at Hospital Discharge. Resuscitation, 82, 845-852. http://dx.doi.org/10.1016/j.resuscitation.2011.02.028

[7] Herlitz, J., Bång, A., Aune, S., Ekström, L., Lundström, G. and Holmberg, S. (2001) Characteristics and Outcome among Patients Suffering In-Hospital Cardiac Arrest in Monitored and Non-Monitored Areas. Resuscitation, 48, 125135. http://dx.doi.org/10.1016/S0300-9572(00)00249-5

[8] Ebell, M.H. and Afonso, A.M. (2011) Pre-Arrest Predictors of Failure to Survive after In-Hospital Cardiopulmonary Resuscitation: A Meta-Analysis. Family Practice, 28, 505-515. http://dx.doi.org/10.1093/fampra/cmr023

[9] Hershey, C.O. and Fisher, L. (1982) Why Outcome of Cardiopulmonary Resuscitation in General Wards Is Poor. Lancet, 2, 31-34. http://dx.doi.org/10.1016/S0140-6736(82)92567-3

[10] Hendrick, J.M., Pijls, N.H., van der Werf, T. and Crul, J.F. (1990) Cardiopulmonary Resuscitation on the General Ward: No Category of Patients Should Be Excluded in Advance. Resuscitation, 20, 163-171. http://dx.doi.org/10.1016/0300-9572(90)90051-F

[11] Sandroni, C., Ferro, G., Santangelo, S., Tortora, F., Mistura, L., Cavallaro, F., Caricato, A. and Antonelli, M. (2004) In-Hospital Cardiac Arrest: Survival Depends Mainly on the Effectiveness of the Emergency Response. Resuscitation, 62, 291-297. http://dx.doi.org/10.1016/j.resuscitation.2004.03.020

[12] Jacobs, I., Nadkarni, V., Bahr, J., Berg, R.A., Billi, J.E., Bossaert, L., Cassan, P., Coovadia, A., D’Este, K., Finn, J., Halperin, H., Handley, A., Herlitz, J., Hickey, R., Idris, A., Kloeck, W., Larkin, G.L., Mancini, M.E., Mason, P., Mears, G., Monsieurs, K., Montgomery, W., Morley, P.G., Nolan, J., Okada, K., Perlman, J., Shuster, M., Steen, P.A., Sterz, F., Tibballs, J., Timerman, S., Truitt, T. and Zideman, D. (2004) Cardiac Arrest and Cardiopulmonary Resuscitation Outcome Reports: Update and Simplification of the Utstein Templates for Resuscitation Registries: A Statement for Healthcare Professionals from a Task Force of the International Liaison Committee on Resuscitation. Circulation, 110, 3385-3397. http://dx.doi.org/10.1161/01.CIR.0000147236.85306.15

[13] Hillman, K.M., Bristow, P.J., Chey, T., Daffurn, K., Jacques, T., Norman, S.L., Bishop, G.F. and Simmons, G. (2001) Antecedents to Hospital Deaths. Internal Medicine Journal, 31, 343-348. http://dx.doi.org/10.1046/j.1445-5994.2001.00077.x

[14] Charlson, M.E., Pompei, P., Ales, K.L. and MacKenzie, C.R. (1987) A New Method of Classifying Prognostic Comorbidity in Longitudinal Studies: Development and Validation. Journal of Chronic Diseases, 40, 373-383. http://dx.doi.org/10.1016/0021-9681(87)90171-8

[15] de Groot, V., Beckerman, H., Lankhorst, G.J. and Bouter, L.M. (2003) How to Measure Comorbidity. A Critical Review of Available Methods. Journal of Clinical Epidemiology, 56, 221-229. http://dx.doi.org/10.1016/S0895-4356(02)00585-1

[16] de Vos, R., Koster, R.W., De Haan, R.J., Oosting, H., van der Wouw, P.A. and Lampe-Schoenmaeckers, A.J. (1999) In-Hospital Cardiopulmonary Resuscitation: Prearrest Morbidity and Outcome. JAMA Internal Medicine, 159, 845-850. http://dx.doi.org/10.1001/archinte.159.8.845

[17] Ballew, K.A., Philbrick, J.T., Caven, D.E. and Schorling, J.B. (1994) Predictors of Survival Following In-Hospital Cardiopulmonary Resuscitation. JAMA Internal Medicine, 154, 2426-2432. 
http://dx.doi.org/10.1001/archinte.1994.00420210060007

[18] Herlitz, J., Rundqvist, S., Bang, A., Aune, S., Lundström, G., Ekström, L. and Lindkvist, J. (2001) Is There a Difference between Women and Men in Characteristics and Outcome after in Hospital Cardiac Arrest? Resuscitation, 49, 15-23. http://dx.doi.org/10.1016/S0300-9572(00)00342-7

[19] Nishiuchi, T., Hiraide, A., Hayashi, Y., Uejima, T., Morita, H., Yukioka, H., Shigemoto, T., Ikeuchi, H., Matsusaka, M., Iwami, T., Shinya, H. and Yokota, J. (2003) Incidence and Survival Rate of Bystander-Witnessed Out-of-Hospital Cardiac Arrest with Cardiac Etiology in Osaka, Japan: A Population-Based Study According to the Utstein Style. Resuscitation, 59, 329-335. http://dx.doi.org/10.1016/S0300-9572(03)00241-7

[20] Ornato, J.P., Peberdy, M.A., Reid, R.D., Feeser, V.R. and Dhindsa, H.S. (2012) Impact of Resuscitation System Errors on Survival from In-Hospital Cardiac Arrest. Resuscitation, 83, 63-69. http://dx.doi.org/10.1016/j.resuscitation.2011.09.009

[21] Franklin, C. and Mathew, J. (1994) Developing Strategies to Prevent in Hospital Cardiac Arrest: Analyzing Responses of Physicians and Nurses in the Hours before the Event. Critical Care Medicine, 22, 244-247. http://dx.doi.org/10.1097/00003246-199402000-00014

[22] Schein, R.M., Hazday, N., Pena, M., Ruben, B.H. and Sprung, C.L. (1990) Clinical Antecedents to In-Hospital Cardiopulmonary Arrest. Chest, 98, 1388-1392. http://dx.doi.org/10.1378/chest.98.6.1388 
Scientific Research Publishing (SCIRP) is one of the largest Open Access journal publishers. It is currently publishing more than 200 open access, online, peer-reviewed journals covering a wide range of academic disciplines. SCIRP serves the worldwide academic communities and contributes to the progress and application of science with its publication.

Other selected journals from SCIRP are listed as below. Submit your manuscript to us via either submit@scirp.org or Online Submission Portal.
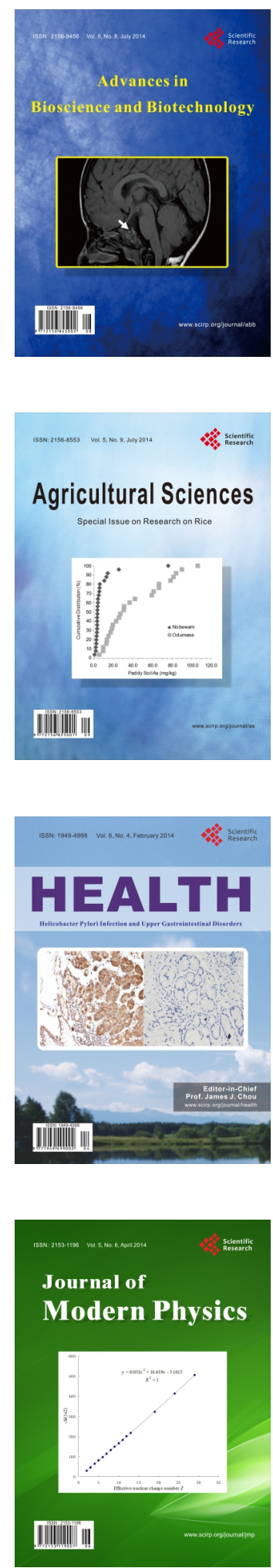
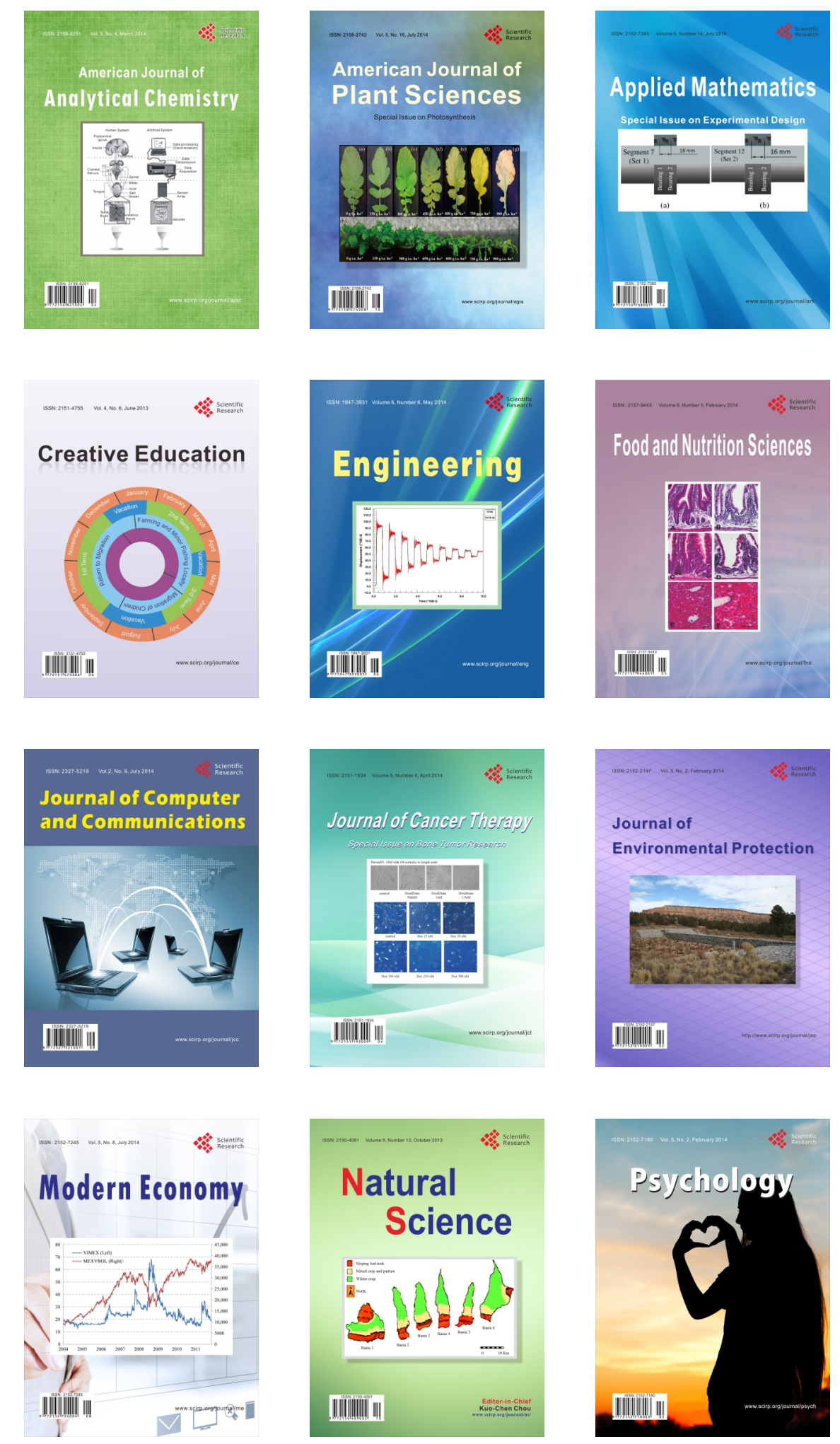